\title{
Larva currens seul signe pathognomonique de la strongyloïdose
}

\author{
par L.-C. BRUMPT et HO THI SANG \\ Institut de Médecine tropicale, 15, rue de l'Ecole de Médecine, F 75006 Paris \\ et C.H.U. Necker-Enfants Malades, 156, rue de Vaugirard, F 75730 Paris
}

\section{Résumé}

L'urticaire linéaire à progression rapide, ou Larva currens, est un symptôme pathognomonique de la strongyloüdose intestinale.

Sa fréquence est dans l'ensemble sous-estimée; elle s'observe électivement dans les infestations d'origine asiatique et africaine et plus rarement dans celles contractées en Amérique.

Symptôme relativement tardif de la strongylö̈dose, Larva currens se voit surtout dans la race blanche. Elle débute plus souvent après le retour des tropiques que dans les pays d'endémie.

L'urticaire linéaire peut être interprétée comme une réaction allergique due à la migration intradermique d'une larve filariforme chez un sujet sensibilisé par la présence de Strongyloides stercoralis.

L'analyse clinique de l'éruption fait penser que ces larves erratiques procèdent d'un mécanisme d'auto-endoinfestation.

\section{Summary}

Larva currens, creeping eruption, or linear urticaria is a pathognomonic symptom of intestinal Strongyloïdiasis.

Its incidence, generally underestimated, is higher with the african and asiatic than with the american strains of Strongyloides stercoralis.

The symptoms, more frequent in the caucasian race, appear relatively late in the course of the infection, very often after the return to temperate climates. 
Larva currens is an allergic reaction due to the intradermic migration of the filariform larva. The clinical aspects are in favor of auto-endo-infestation and in no case of auto-exoinfestation.

Et depuis quarante ans, si ce n'est davantage, Cet homme vivait...

Georges Fourest, Le Géranium ovipare, Une vie.

L'auto-infestation assure à la strongyloïdose une longévité sans limites. Une de nos malades en fut atteinte pendant 71 ans. Cette parasitose est le plus souvent bénigne mais elle peut devenir grave, voire mortelle si le porteur de Strongylö̈des reçoit occasionnellement des corticostéroïdes ou des immuno-suppresseurs.

La recherche systématique de Strongyloides stercoralis dans les pays tropicaux ou chez des transplantés originaires des tropiques montre à la fois la fréquence de la parasitose et sa latence. Les signes digestifs n'ont rien de spécifique et manquent neuf fois sur dix. Par contre, il existe souvent un symptôme dermatologique, l'urticaire linéaire ou larva currens qui est pathognomonique de la strongyloïdose intestinale.

Parmi de nombreuses observations, nous en citerons trois.

Obs. 1 .

M. G..., âgé de 65 ans, dessinateur industriel, demande le 14 mai 1965 par téléphone un conseil pour une filariose contractée au Gabon quarante ans auparavant. Comme cette longévité est triple de la normale pour une Loa loa, nous insistons pour voir ce malade.

Il a passé deux ans au Gabon, de 1925 à 1927, dans une exploitation forestière. Mécanicien, il posait des rails dans la forêt et réparait les remorqueurs fluviaux sur l'Ogoué. Il n'a souffert ni de paludisme, ni d'amibiase et n'a consulté le Docteur Schweitzer que pour des troubles mineurs.

Il revient définitivement en France en 1927. Un an après son retour, il consulte à l'hôpital Saint-Louis pour du prurit. Des ankylostomes sont décelés qui mettent trois ans à disparaître, le vermifuge utilisé étant la fougère mâle.

Les signes cutanés persistent; le malade consulte de nombreux médecins, récolte au passage des prescriptions antipaludiques et antiamibiennes. Il épuise toutes les ressources de l'allopathie et de l'homéopathie quand arrive la guerre; il est mobilisé, en 1939, à Beauvais, où il prend contact avec de nombreux médecins qui l'introduisent auprès de tropicalistes; le diagnostic de filariose est établi d'après les signes cliniques, l'hyperéosinophilie et une intradermoréaction. Il est traité par les arsenicaux et les antimoniés jusqu'en 1948, date à laquelle il bénéficie des premiers échantillons de diéthyl-carbamazine qui devait être diffusée ultérieurement sous le nom de Notézine. Le malade en fait des cures répétées sans la moindre amélioration des manifestations prurigineuses qui le harcèlent jour et nuit. Dans l'atelier qu'il dirige, il se gratte le dos au chambranle des portes sous les regards admiratifs des apprentis, pleins d'égards pour leur ancien «qui a fait la colonie ». L'échec de la Notézine fait mettre en doute le diagnostic de loase qui est remplacé par celui de \& larbich ». Le malade se livre alors aux frictions de pommade au Gammexane et aux pulvérisations de Kélène avec un insuccès persistant.

Conscient du caractère extraordinaire de son cas, il rédige pendant neuf ans, d'avril 
1954 à octobre 1963, une auto-observation et note 2078 émergences cutanées en plus des jours où il écrit: «Partout! Intenable!»

Nous sommes persuadés qu'il a bien souffert de loase; on ne flotte pas impunément des billes d'okoumé sur l'Ogoué sans contracter cette filariose dont il décrit vers 1939 une manifestation oculaire typique; mais la filariose a eu trois fois le temps de guérir spontanément. Actuellement le diagnostic de strongylö̈dose cutanée s'impose et se confirme par une légère hyperéosinophilie à $9 \%$ et la présence d'anguillules dans les selles.

Notre proposition de guérir $M$. G... en un jour le laisse complètement sceptique ; il nous répond que son calendrier thérapeutique ne comporte aucun créneau; la semaine est divisée en deux jours de traitement contre les hématozoaires, deux jours contre les amibes et trois jours contre les filaires. De plus, c'est l'époque où il fait sa cure mensuelle de Streptomycine; enfin, il a pris rendez-vous avec un chirurgien pour une cure herniaire.

Sans avoir bénéficié au temps de nos études d'un enseignement de la psychologie, nous comprenons que le malade n'est pas préparé à la guérison. Faut-il même le guérir ? Jules Romains n'a-t-il pas écrit que «chacun doit trouver la maladie qui lui convient et qui l'aide à vivre»? Nous décidons pourtant de le traiter et lui remettons négligemment quelques comprimés de Thiabendazole à prendre avant l'intervention chirurgicale. Au cours de sa convalescence, il revient nous voir; son scepticisme a été ébranlé par une sédation inhabituellement longue des symptômes cutanés suivie cependant d'une rechute. Le Thiabendazole est prescrit aux doses de 50 milligrammes $/ \mathrm{kg}$ deux jours consécutifs. Il guérit définitivement de sa strongylö̈dose mais non de sa psychasthénie. En 1972, il est encore obsédé par le paludisme et l'amibiase dont il n'a jamais eu le moindre symptôme.

\section{Obs. 2.}

M. L..., âgé de 35 ans, instituteur, a enseigné deux ans au Tchad et huit ans au Congo. Il revient en France en 1955. Trois ans après son retour, en 1958, il voit apparaître sur la peau des traînées rouges serpigineuses et prurigineuses. Les émergences se produisent le plus souvent dans la région lombo-sacrée qu'il surnomme la « Mer des Sargasses». L'urticaire linéaire évolue capricieusement, précédée souvent de troubles digestifs et, se répète pendant une quinzaine de jours avec des périodes d'accalmie de même durée. Il consulte des tropicalistes. Le diagnostic de filariose aboutit à des cures de Notézine avec un insuccès constant. Le diagnostic de rechange est \&larbich » et la pommade au Gammexane à $1 / 10^{\circ}$ est prescrite. Le malade se soigne avec assiduité et utilise 1500 grammes de pommade soit 150 grammes d'hexachloro-cyclo-hexane.

Les turbulences urticariennes continuent ; de nouveaux symptômes apparaissent : anorexie, nausées, vomissements, amaigrissement important, insomnie, nervosité et tremblement. Un de ses parents, pharmacien, lui conseille d'abandonner l'HCH et de recourir à la cryothérapie. Il lui fournit successivement 75 tubes de $100 \mathrm{ml}$ de chlorure d'éthyle. Mais lorsque le malade attaque ses «bestioles» au Kélène, elles se dispersent dans tous les azimuts, et ne sont pas tuées.

En 1965, l'éosinophilie est de $10 \%$; des larves de Strongyloïdes sont présentes dans les selles. La guérison est obtenue avec une dose de $50 \mathrm{mg} / \mathrm{kg}$ de Thiabendazole.

\section{Obs. 3 .}

M. R..., ingénieur, âgé de 58 ans, consulte en 1960. Il a travaillé à Madagascar en 1928, en Malaisie de 1930 à 1932, en Indochine de 1933 à 1934, au Cameroun de 1938 à 1945 et en Guinée de 1950 à 1959. 
C'est en 1932, en Malaisie, qu'il a commencé à souffrir de «filariose». Les filaires, dit-il, sortent sous la peau surtout à la tombée de la nuit et presque toujours par groupe de trois, avec une fréquence de deux ou trois poussées par mois. Il signale bien des troubles digestifs, du météorisme et de la diarrhée, mais il affirme qu'ils n'ont aucune corrélation avec les signes cutanés.

D'après ses conversations avec les forestiers du Gabon, il sait qu'il ne s'agit pas de loase mais d'une filaire très différente contractée en Malaisie; il refuse le diagnostic de strongyloïdose cutanée et disparaît pour quelques années. Il reparaît en 1963 et n'accepte pas de traiter sa maladie au prix de vingt jours de traitement par la Dithiazanine. En décembre 1965, il écrit de sa retraite provinciale. Les manifestations prurigineuses se sont aggravées et, fait nouveau, les «filaires» persistent même pendant la journée ; il voudrait en profiter pour faire extirper une des trois filaires aux fins de détermination parasitologique.

Nous répondons que ce n'est plus en 21 jours de traitement mais un jour qu'il peut être guéri ; il consent enfin à un examen coprologique et guérit définitivement avec $50 \mathrm{mg} / \mathrm{kg}$ de Thiabendazole deux jours consécutifs.

\section{Historique}

Ces manifestations cutanées de la Strongyloïdose sont connues depuis longtemps.

Dès 1926, elles ont été décrites par Fülleborn qui en observa huit cas dont le sien sur dix porteurs de Strongyloides stercoralis. Il pensait que la lésion partait de l'anus pour irradier ensuite et interprétait le fait comme une auto-exoinfestation.

Napier (1949) et Caplan (1949) en ont observé de très nombreux cas chez des prisonniers libérés des camps japonais du Sud-Est Asiatique, de Birmanie et de Malaisie.

Sandosham (1952) à Singapour constata que le phénomène s'observait électıvement chez des Européens lorsqu'ils quittaient la Malaisie. Il pensait qu'il s'agissait peut-être de souches de Strongyloïdes d'animaux.

Galliard et Chabaud (1952) étudièrent expérimentalement une souche isolée chez un malade de Riou. Ils trouvèrent lors de la première coproculture des anomalies de taille qui ne se maintinrent pas après passage expérimental chez le chien.

Arthur et Shelley (1958), à propos d'une observation très discutable cliniquement, proposèrent le nom imagé de Larva currens, qui mérite de prévaloir.

M.-V. Riou (1962) nous fit bien connaître cette urticaire linéaire observée après leur rapatriement chez des porteurs de Strongylö̈des qu'il avait suivis en Indochine, puis en France.

\section{Description clinique des ćléments}

Le début est brusque : le malade ressent une sensation de prurit, regarde immédiatement sa peau et constate une papule urticarienne qui parfois a déjà un aspect allongé. 
La morphologie est typique en moins d'une heure.

L'élément urticarien a une longueur moyenne de 10 centimètres, parfois 20 ou 30 , et une largeur de 2 à 3 millimètres. Il comprend deux parties : la zone antérieure de progression qui est un bourrelet rouge vif surélevé, doux au toucher et la zone postérieure plus ancienne qui est affaissée, un peu moins rouge et s'effaçant aussi à la pression.

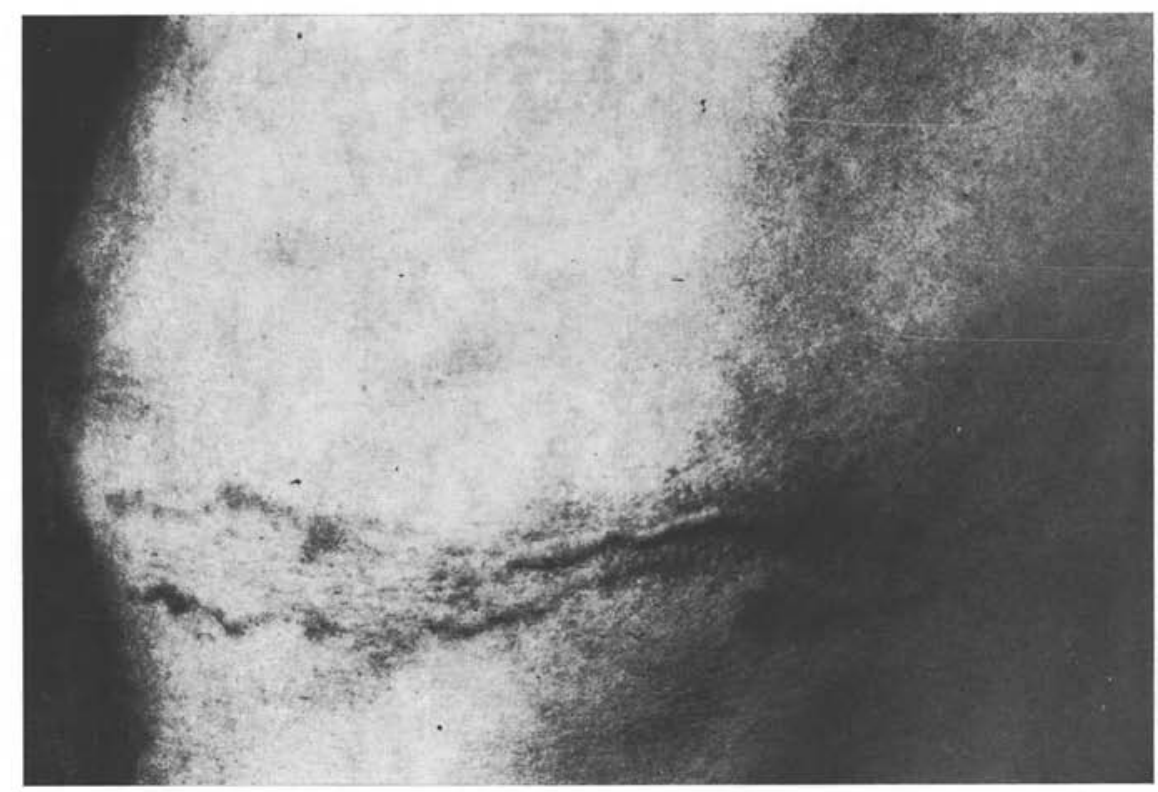

Fig. 1. - Deux larves se déplacent parallèlement à la ceinture

L'urticaire linéaire peut revêtir trois aspects, rectiligne, sinueux ou lové, qui correspondent souvent à trois émergences successives de la même larve séparées par des intervalles de peau normale, dues sans doute à des plongées en profondeur dans le derme. Ceci explique l'interprétation du malade $\mathrm{n}^{\circ} 3$ sur le nombre de ses parasites.

La vitesse de progression rapide au début, puis plus lente est de 2 à $10 \mathrm{~cm}$ à l'heure, ce qui justifie la dénomination de larva currens par comparaison avec d'autres dermites linéaires ou creeping disease.

La durée de la manifestation est de 12 à 48 heures.

La disparition est complète sans desquamation ni pigmentation.

L'horaire des émergences se place souvent entre 18 heures et minuit, ce qui explique le désarroi du malade qui n'est jamais certain de montrer sa peau en temps voulu au médecin ou au dermatologue. 
Le rythme des émergences est parfois discontinu par salves de quelques jours avec des rémissions d'une à trois semaines ; mais, chez d'autres malades les éruptions sont permanentes sans tendance à l'amélioration.

Dans la peau, le niveau de la lésion est sous-épidermique. Une cicatrice d'intervention chirurgicale est un obstacle infranchissable. La légère striction due à la ceinture d'un sous-vêtement imprime aux larves un trajet horizontal (fig. 1).

Le nombre maximum des lésions observées simultanément fut de 10 à 20 chez un légionnaire contaminé en Indochine.

La topographie de l'éruption est très caractéristique. Le schéma (fig. 2), ci-contre, correspond aux émergences notées dans l'observation $\mathrm{n}^{\circ} 1$, pendant 9 ans.

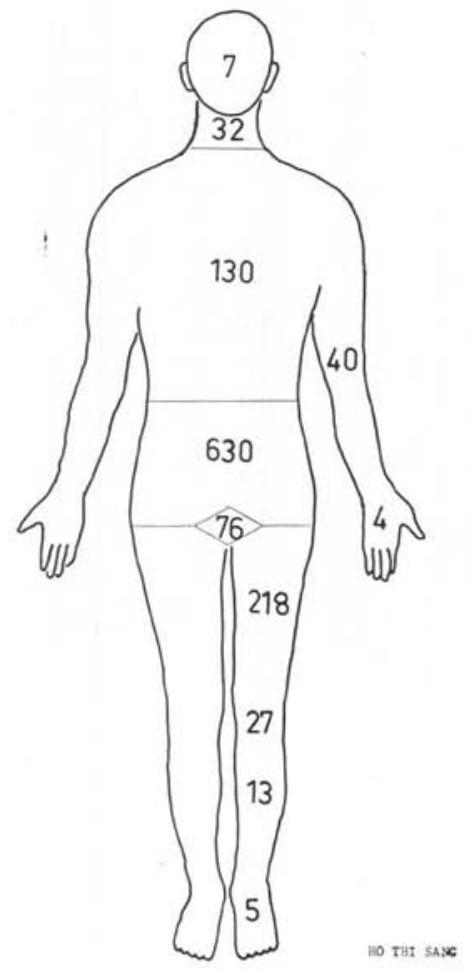

De haut en bas :

paupière 1 ,

oreille 2 ,

joue et région maxillaire 4 ,

cou et nuque 32 ,

thorax 130 ,

abdomen, hanches, lombes, fesses 630 ,

cuisses 218 ,

genou 27 ,

mollet 13,

pieds, chevilles 5 .

Il faut ajouter une extension vers les membres supérieurs: bras et avant-bras 40 avec une prédominance gauche.

main gauche 4 .

Les localisations périnéales se décomposent en : région péri-anale 28 ,

périnée 29 ,

pénis 15 ,

scrotum 3,

prépuce 1 .

\section{Signes d'accompagnement}

Le prurit constant est d'intensité variable ; certains sujets sont réveillés pendant la nuit ; il est rare que le prurit s'accompagne de lésions de grattage ou se complique d'eczéma ou de lichénification. Napier (1949) note avec une grande justesse que le prurit n'est pas le motif de consultation; il est signalé occasionnellement au cours 
de la conversation. Depuis que nous la recherchons systématiquement par l'interrogatoire, nous trouvons beaucoup plus fréquemment l'urticaire linéaire.

Il n'existe ni fièvre, ni adénopathies satellites, ni autres signes d'allergie cutanée ou respiratoire. L'exacerbation des signes digestifs, quand ils sont présents, est souvent le prélude d'éruptions serpigineuses.

Le retentissement psychique variable selon le terrain est très explicable par le caractère récidivant et tenace de ce symptôme mystérieux qui reste sans diagnostic et sans traitement pendant de nombreuses années.

L'éosinophilie sanguine peut être élevée comme dans la plupart des strongyloïdoses ; mais dans les infestations très anciennes nous avons souvent noté une éosinophilie subnormale ou même normale.

Les résultats de l'examen coprologique sont sans parallélisme avec l'importance des signes cutanés; certains de nos malades sont pensionnés pour amibiase et ont fait l'objet de dix ou quinze examens coprologiques sans que la présence des anguillules ait été signalée. Souvent les larves sont peu nombreuses et décelables seulement par la technique de Baermann.

\section{Causes favorisantes}

L'origine géographique des souches est d'une très grande importance. Déjà Caplan, Napier, Sandosham insistèrent sur la contamination des malades dans le Sud-Est Asiatique. Nous voyons, en effet, les Larva currens avec une très grande fréquence dans nos observations asiatiques et africaines alors que le symptôme est exceptionnel dans les cas américains de strongyloïdose ; ainsi nous n'avons noté qu'un cas brésilien chez un agriculteur italien, un cas colombien et trois cas antillais sur plusieurs centaines de porteurs de strongylö̈des. Larva currens ne fait pas partie des descriptions cliniques de strongyloïdose des auteurs Sud-Américains.

Les sujets de race blanche sont beaucoup plus souvent atteints. Nous n'avons observé que trois cas chez des mulâtres et un cas chez un pur Africain sous forme d'un bourrelet noir et doux au toucher. Sandosham n'en observait que chez les Européens de Singapour. Nous n'avons pas vu de Larva currens chez des Chinois, des Laotiens, des Vietnamiens ou des Indiens; mais un médecin cambodgien atteint luimême de Larva currens en a vu chez ses compatriotes.

En plus de la prédominance raciale, il existe sans doute une prédisposition individuelle; dans un cas d'infestation volontaire, le receveur a présenté de l'urticaire linéaire alors que le donneur ne l'avait pas signalé.

Nous ne pensons pas que l'âge et le sexe influent sur la fréquence du symptôme bien que nos dossiers concernent surtout des hommes adultes.

L'ancienneté de la strongylö̈dose est habituelle. Les signes cutanés peuvent n'apparaître qu'au bout de dix ans ; une fois, nous les avons observés au bout de six mois d'une infestation volontaire; mais il faut reconnaître que la virulence avait été sti. mulée par une corticothérapie occasionnelle. 
Chez les Européens, les symptômes débutent rarement aux tropiques mais souvent après le rapatriement, fait qui a été bien noté par Caplan, Napier, Sandosham et Riou chez ses malades d'Indochine.

La chaleur du lit, l'activité physique, l'effort, la sudation stimulent les émergences. Le malade $\mathrm{n}^{\circ} 1$ vit apparaitre les larves «dans les parties du corps qui travaillent ». Un aviateur présente de l'urticaire dans les avions non pressurisés et pas dans les avions à réaction. Un médecin asiatique prévoit à coup sûr la pluie comme si la dépression barométrique favorisait l'issue des larves.

Les poussées d'urticaire sont souvent provoquées par l'administration d'un vermifuge irritant pour les strongyloïdes de l'intestin comme le bismuth, la notézine, le mébendazole. Une cure manquée de Thiabendazole est parfois suivie à brève échéance d'une reprise de l'urticaire. Ces souches sont particulièrement résistantes à la thérapeutique et nous prescrivons souvent le Thiabendazole deux ou trois jours consécutifs aux doses de $50 \mathrm{mg} / \mathrm{kg}$.

\section{Pathogénie}

L'urticaire linéaire a été expliquée par de nombreux auteurs comme une réaction allergique; il ne s'agit pas d'allergie à distance comme l'urticaire localisée ou généralisée que l'on observe surtout dans les primo-infections à strongyloïdes, mais d'une réaction allergique due à la présence d'une larve dans un épiderme sensibilisé.

Nitzulesco a réussi, en injectant une solution de résoquine en avant de la lésion, à provoquer un demi-tour de la larve. De même les jets de Kélène et les frictions au gammexane déterminent la dispersion des larves.

Il est probable qu'il s'agit d'une larve au stade strongyloïde infestant. La biopsie cutanée, déjà difficile dans la creeping disease, où la larve d'Ancylostoma brasiliense se déplace lentement, serait ici bien hasardeuse.

L'origine et le trajet des larves font l'objet de deux théories : l'auto-exoinfestation et l'auto-endoinfestation.

La première est défendue par la plupart des auteurs, en particulier Fülleborn, qui fait partir la lésion cutanée de l'anus; dans ce cas, il s'agirait de larves issues d'une sorte de coproculture qui se situerait dans les plis radiés de l'anus à partir de parcelles de matières fécales. Cet auteur et d'autres ensuite, tout en préconisant une hygiène anale minutieuse, reconnaissent qu'elle n'influe pas sur la fréquence des poussées d'urticaire.

Nitzulescu est le premier à insister nettement sur le fait que l'urticaire ne venait pas de l'anus, mais émergeait loin de là.

Nous n'approuvons pas le mécanisme de l'auto-exoinfestation pour les raisons suivantes :

a) le prurit anal n'est pas un symptôme habituel des strongyloïdoses, qu'elles soient ou non accompagnées d'urticaire linéaire. Ce qui facilite notre interprétation clinique ; 
b) jamais nos nombreux malades n'ont présenté de lésions à point de départ anal ;

c) sur un grand nombre de localisations comme dans l'observation $\mathrm{n}^{\circ} 1$, un petit nombre de larves émergent au niveau du périnée de la même façon qu'en tout autre point du corps et sans contiguïté anale.

Nous croyons davantage à l'auto-endoinfestation. Hartz (1947), dans une étude histologique, a montré que de nombreuses larves filariformes infestantes pouvaient traverser toute la paroi du côlon.

Nous pensons que certaines larves gagnent les vaisseaux veineux ou lymphatiques et peuvent terminer leur cycle évolutif ; mais un certain nombre de larves erratiques sont très capables de cheminer de proche en proche à travers le péritoine et la paroi abdominale pour émerger sous la peau. Elles sont destinées à mourir en 48 heures environ; elles restent mobiles au-delà du traitement bref par le Thiabendazole, mais ne rejoignent pas ultérieurement les poumons.

L'origine sigmoïdienne des larves est probable si l'on en juge par la prédominance antérieure abdominale gauche des émergences.

Quant aux localisations lointaines comme celles du bras, elles ne peuvent être expliquées que par une dissémination vasculaire.

\section{Souches humaines ou souches animales}

Des éruptions serpigineuses ont été reproduites chez l'homme en utilisant des strongyloïdes d'animaux, ainsi Little en expérimentant avec S. myopotami et S. procyonis de la loutre et du raton-laveur. Sandosham avait émis l'hypothèse que les strongyloïdoses compliquées d'urticaire linéaire provenaient d'espèces animales. Galliard et Chabaud, à partir de coprocultures provenant d'un cas clinique de Larva currens, ont obtenu des spécimens femelles et mâles de taille double de la normale ; de plus, les femelles faisaient preuve d'une plus grande fécondité, mais ces caractères ne se montrèrent pas stables après plusieurs passages chez le chien.

En somme, les cas de Larva currens semblent bien correspondre à des souches humaines de Strongyloides stercoralis.

\section{Diagnostic différentiel}

D'après nos observations, deux diagnostics sont souvent portés dans les cas d'urticaire linéaire : filariose et larbich. Lorsque le sujet a été contaminé en Afrique équatoriale, on pense à la loase. Mais les manifestations oculaires et les œdèmes de Calabar sont bien caractéristiques même si les microfilaires ne sont pas décelées dans le sang; lorsque la macrofilaire s'installe sous la peau à l'occasion d'une cure de Notézine, elle est visible et palpable sous l'aspect d'un bourrelet blanchâtre; elle est même extirpable. 
Le diagnostic le plus souvent posé est celui de "larbich », nom vernaculaire africain de la dermite linéaire serpigineuse ou «creeping disease» déterminée par la larve au $3^{\circ}$ stade de Ancylostoma braziliense, parasite des chiens, cosmopolite à prédominance tropicale.

Les lésions siègent sur les parties du corps qui ont été en contact avec le sable humide des plages tropicales, c'est-à-dire souvent au pied. L'incubation courte est de 2 à 8 jours; le bourrelet érythémateux correspond à un parasite profondément enchassé dans la peau, il se déplace lentement, à la vitesse de un à deux centimètres par jour, persiste de façon continue pendant deux ou trois mois et guérit spontanément. La partie postérieure de la lésion est le siège d'une desquamation et de pigmentation; le prurit beaucoup plus intense que dans la strongyloïdose conduit au grattage et à la surinfection des lésions. Nous avons toujours été déçus par les frictions de pommade au gammexane; par contre, la cryothérapie au Kélène appliquée au pôle d'accroissement de la lésion est très efficace.

Il faut reconnaître que le Thiabendazole a le mérite de guérir le larbich avec $70 \%$ d'efficacité, alors que la strongyloïdose est guérie à 90 ou $95 \%$.

\section{Bibliographie}

1. Arthur (R. P.) et Shelley (W. B.), 1958. - Larva currens. A distinctive variant of Cutaneous Larva Migrans due to Strongyloides stercoralis. A.M.A. Archives of Dermatol., 78, 180-190.

2. Caplan (J.P.), 1949. - Creeping eruption and intestinal Strongyloidiasis. Brit. Med. Jour., 1, 396.

3. Fulleborn (F.), 1926. - Hautquaddeln und Autoinfektion bei Strongyloidesträgern. Arch. f. Schiffs. u. Trop. Hyg., 30, 731-732.

4. HARTZ (P.H.), 1946. - Human Strongyloidiasis with internal auto-infection. Arch. Pathology, 41, 601-611.

5. Litrue (L. D.), 1965. - Dermatitis in a human volunteer infected with Strongyloides of Nutria and Raccoon. Am. J. Trop. Med. et Hyg., 14, 1007-1009.

6. NAPIER (L. E.), 1949. - Strongyloidiasis among ex prisoners of War. Jour. Trop. Med. Hyg., 52, 46-48.

7. Nitzulescu (V.), 1967. - Sur un cas de Strongylö̈dose à dermatite linéaire. Bull. Soc. Path. exot., 60, 563-568.

8. Riou (M. V.), 1962. - Hyperéosinophilie sanguine et urticaire linéaire au cours de certaines anguilluloses. Bull. Soc. Path. exot., 55, 716-719.

9. Sandosham (A. A.), 1952. - Creeping eruption with Strongyloides stercoralis infection contracted in the Far-East. J. of Helminth., 26, 1-24. 